\title{
CETACEANS AND FISHERY INTERACTIONS ALONG THE ESPÍRITO SANTO STATE, SOUTHEASTERN BRAZIL DURING 1994-2001
}

\author{
Ricardo de Freitas Netto ${ }^{1, *}$ and Lupércio Araújo Barbosa ${ }^{1}$
}

The incidental capture of cetaceans in passive fishing gears such as gillnets represents the major threat to their conservation (Perrin et al., 1994). Along the Brazilian coast the franciscana, Pontoporia blainvillei, and marine tucuxi, Sotalia fluviatilis, are the most vulnerable species. Confined to coastal waters, they are often incidentally caught in fisheries (e.g. Siciliano, 1994; Secchi et al., 1997; Di Beneditto et al., 1998; IBAMA, 2001; Ott et al. 2002). Information on the occurrence of cetaceans along the Espírito Santo coast, southeastern Brazil, is limited to a few reports of species incidentally caught during fishing activities (e.g. Di Beneditto et al., 1990; Siciliano, 1994), stranded on beaches (e.g. Barros, 1991; Borobia et al., 1991; Gasparini and Sazima, 1996; Barros et al., 1997a,b; Barbosa et al., 2000) or occasionally sighted (e.g. Lodi et al., 1996). The objective of this report is to provide new data on the incidental capture of cetaceans along the Espírito Santo coast from 1994 to 2001 and to compile previous information for this region. Recent data on the incidental captures were obtained opportunistically by collecting stranded carcasses along the coast with indicative marks of interactions with fisheries (e.g. wounds, scars and fishing gear fragments on the animals' body) (Perrin et al., 1994). One stranding report published by the local media was also included. Species identification, sex and standard length of the animals, as well as locality and date of stranding were recorded. The skeletons of all small cetaceans were deposited at the ORCA collection (Table 1).

Between 1994 and 2001 twelve stranded cetaceans with wounds that indicate interaction with fishery were collected along the Espírito Santo coast. The highest number of specimens was observed for S. fluviatilis $(\mathrm{n}=8)$, followed by the rough-toothed dolphin (Steno bredanensis) $(\mathrm{n}=3)$ and the humpback whale (Megaptera novaeangliae) $(\mathrm{n}=1)$ (Table1). When reviewing the information on cetacean and fishery interactions in Brazil, Siciliano (1994) reported the stranding and incidental catch of 44 specimens along the Espírito Santo coast between 1983 and 1994. During that period, S. fluviatilis was also the species most commonly found $(\mathrm{n}=28)$, followed by P. blainvillei $(\mathrm{n}=13), M$. novaeangliae $(\mathrm{n}=1)$, Tursiops truncatus $(\mathrm{n}=1)$ and Physeter macrocephalus $(\mathrm{n}=1)$.

The coastal habitat of $S$. fluviatilis is likely the main factor that makes the species more vulnerable to incidental captures in the area. The lower number of animals reported in this study in relation to the previous period (1983 to 1994) compiled by Siciliano
(1994) is probably due to a lower monitoring effort of the fishing villages and beaches rather than a marked decline in the by-catch or any change in the abundance of the species. Although Barros (1991) reported the incidental catch of a female tucuxi in the region, all individuals of known gender in the present study were males. Though sample sizes are small, this could suggest a higher vulnerability of male tucuxis to fishing activities. Assuming that Espírito Santo tucuxis exhibit similar growth patterns as those from adjacent northern Rio de Janeiro waters, where animals reach maturity at $180 \mathrm{~cm}$ in length (Di Beneditto et. al., 2001), at least five specimens in this study can be considered adults (see Table 1).

Although this study presents the first indications of incidental take of rough-toothed dolphins along the Espírito Santo coast, several other captures of this species have been reported elsewhere along the Brazilian coast (e.g. Siciliano 1994, Lodi and Hetzel, 1998, Di Beneditto et al., 2001). The lower ratio of $S$. bredanensis versus $S$. fluviatilis observed in this study might be partly due to the relatively low abundance of rough-tooth-dolphins in coastal areas off Espírito Santo, and therefore may be less vulnerable to coastal fishing activities. Dedicated surveys are needed to address this question.

The incidental capture of a humpback whale in fishing gears along the Espírito Santo coast has been previously reported by Barros (1991). Other cases of fishery interactions involving this species, especially juveniles, have also been observed in southeastern and southern Brazil (e.g. Siciliano, 1987, Pizzorno et al., 1998, Danilewicz et al., 2002) and may represent an increasing threat to the recovery of the species.

Although interactions of $P$. blainvillei with fisheries have been reported previously for this area (Di Beneditto et. al., 1990; Siciliano, 1994), none was recorded during this study. Despite some logistical constrains, the lack of any record of the species in the region in the last few years was unexpected. Considering that the Espírito Santo coast represents the northernmost limit for franciscanas and possibly contain an isolated population (Ramos et al., 2002; Secchi et al., 2002, Siciliano et al., 2002), a better understanding of the current distribution and abundance of the species in the region is particularly important.

The present number of cetaceans incidentally caught and recorded along the Espírito Santo coast probably represents an underestimation of the problem in the

\footnotetext{
${ }^{1}$ Organização Consciência Ambiental (ORCA). Rua São Paulo, 21, Praia da Costa, Vila Velha, ES, 29101-300, Brazil.

- Corresponding author: Rua Celso Calmon, 445, Praia do Canto, Vitória, ES, 29055-590, Brazil.

E-mail: nettoricardo@hotmail.com.
} 
Table 1. Records of cetaceans stranded along the Espírito Santo coast presenting wounds that may suggest interactions with fisheries

\begin{tabular}{|c|c|c|c|c|c|c|}
\hline $\begin{array}{l}\text { SPECIES } \\
\text { (COLLECTION NUMBER) }\end{array}$ & $\mathrm{N}^{\mathrm{o}}$ & SEX & $\begin{array}{c}\text { TOTAL } \\
\text { LENGTH }(\mathrm{cm})\end{array}$ & LOCAL & $\begin{array}{l}\text { GEOGRAPHIC } \\
\text { COORDINATES }\end{array}$ & DATE \\
\hline Megaptera novaeangliae ${ }^{1 ; 2}$ & 1 & 오 & 490 & $\begin{array}{l}\text { Ponta da Fruta - Vila } \\
\text { Velha }\end{array}$ & $20^{\circ} 31^{\prime} \mathrm{S} ; 40^{\circ} 22^{\prime} \mathrm{W}$ & 28 October 1987 \\
\hline Megaptera novaeangliae (MN008) & 1 & - & 700 & $\begin{array}{l}\text { Pontal do Ipiranga - } \\
\text { Linhares }\end{array}$ & $19^{\circ} 04^{\prime} \mathrm{S} ; 39^{\circ} 44^{\prime} \mathrm{W}$ & 3 November 2000 \\
\hline Physeter macrocephalus ${ }^{2}$ & 1 & - & - & Meaípe - Guarapari & $20^{\circ} 39^{\prime} \mathrm{S} ; 40^{\circ} 27^{\prime} \mathrm{W}$ & 6 August 1981 \\
\hline Pontoporia blainvillei ${ }^{3}$ & 1 & - & - & Regência - Linhares & $19^{\circ} 38^{\prime} \mathrm{S} ; 39^{\circ} 49^{\prime} \mathrm{W}$ & 1987 \\
\hline Pontoporia blainvillei ${ }^{4}$ & 2 & - & - & Regência - Linhares & $19^{\circ} 38^{\prime} \mathrm{S} ; 39^{\circ} 49^{\prime} \mathrm{W}$ & 1987 \\
\hline Pontoporia blainvillei 5 & 5 & - & - & Regência - Linhares & $19^{\circ} 38^{\prime} \mathrm{S} ; 39^{\circ} 49^{\prime} \mathrm{W}$ & February 1989 \\
\hline Pontoporia blainvillei ${ }^{4}$ & 2 & - & - & Regência - Linhares & $19^{\circ} 38^{\prime} \mathrm{S} ; 39^{\circ} 49^{\prime} \mathrm{W}$ & September 1989 \\
\hline Pontoporia blainvillei ${ }^{4}$ & 1 & 우 & - & Itaúnas & $18^{\circ} 25^{\prime} \mathrm{S} ; 30^{\circ} 42^{\prime} \mathrm{W}$ & 12 February 1991 \\
\hline Pontoporia blainvillei ${ }^{4}$ & 1 & 우 & - & Guriri - São Mateus & $18^{\circ} 42^{\prime} \mathrm{S} ; 39^{\circ} 51^{\prime} \mathrm{W}$ & 28 February 1991 \\
\hline Pontoporia blainvillei ${ }^{4}$ & 1 & - & - & Guriri - São Mateus & $18^{\circ} 42^{\prime} \mathrm{S} ; 39^{\circ} 51^{\prime} \mathrm{W}$ & 17 January 1992 \\
\hline Steno bredanensis (SB002) & 1 & - & 245 & $\begin{array}{l}\text { Praia de Itaparica - } \\
\text { Vila Velha }\end{array}$ & $20^{\circ} 21^{\prime} \mathrm{S} ; 40^{\circ} 16^{\prime} \mathrm{W}$ & 5 June 1996 \\
\hline Steno bredanensis (SB004) & 1 & 우 & 270 & $\begin{array}{l}\text { Praia de Setiba - } \\
\text { Guarapari }\end{array}$ & $20^{\circ} 38^{\prime} \mathrm{S} ; 40^{\circ} 26^{\prime} \mathrm{W}$ & 22 May 1999 \\
\hline Steno bredanensis (SB007) & 1 & 우 & 265 & $\begin{array}{l}\text { Porto de Tubarão - } \\
\text { Vitória }\end{array}$ & $20^{\circ} 18^{\prime} \mathrm{S} ; 40^{\circ} 14^{\prime} \mathrm{W}$ & 19 April 2000 \\
\hline Sotalia fluviatilis ${ }^{2}$ & 1 & O & 206 & $\begin{array}{l}\text { Praia de Itapuã - Vila } \\
\text { Velha }\end{array}$ & $20^{\circ} 22^{\prime} \mathrm{S} ; 40^{\circ} 17^{\prime} \mathrm{W}$ & 05 June1983 \\
\hline Sotalia fluviatilis ${ }^{3}$ & 2 & - & - & Regência - Linhares & $19^{\circ} 38^{\prime} \mathrm{S} ; 39^{\circ} 49^{\prime} \mathrm{W}$ & 1987 \\
\hline Sotalia fluviatilis 5 & 6 & - & - & Regência - Linhares & $19^{\circ} 38^{\prime} \mathrm{S} ; 39^{\circ} 49^{\prime} \mathrm{W}$ & $\begin{array}{l}\text { January- } \\
\text { March/1989 }\end{array}$ \\
\hline Sotalia fluviatilis 5 & 3 & - & - & Conceição da Barra & $18^{\circ} 38^{\prime} \mathrm{S} ; 39^{\circ} 51^{\prime} \mathrm{W}$ & March 1989 \\
\hline Sotalia fluviatilis 4 & 4 & - & - & Regência - Linhares & $19^{\circ} 38^{\prime} \mathrm{S} ; 39^{\circ} 49^{\prime} \mathrm{W}$ & 1987-1990 \\
\hline Sotalia fluviatilis 4 & 12 & - & $86-190$ & Guriri - São Mateus & $18^{\circ} 42^{\prime} \mathrm{S} ; 3^{\circ} 51^{\prime} \mathrm{W}$ & $\begin{array}{l}\text { December 1988- } \\
\text { August } 1993\end{array}$ \\
\hline Sotalia fluviatilis (SF003) & 1 & $\sigma^{\prime}$ & 200 & $\begin{array}{l}\text { Praia do Sol - } \\
\text { Guarapari }\end{array}$ & $20^{\circ} 38^{\prime} \mathrm{S} ; 40^{\circ} 26^{\prime} \mathrm{W}$ & 18 May 1994 \\
\hline Sotalia fluviatilis (SF004) & 1 & $\sigma$ & 209 & $\begin{array}{l}\text { Ponta da Fruta - Vila } \\
\text { Velha }\end{array}$ & $20^{\circ} 31^{\prime} \mathrm{S} ; 40^{\circ} 22^{\prime} \mathrm{W}$ & 25 July 1995 \\
\hline Sotalia fluviatilis (SF006) & 1 & 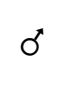 & 222 & $\begin{array}{l}\text { Barra do Jucú - Vila } \\
\text { Velha }\end{array}$ & $20^{\circ} 25^{\prime} \mathrm{S} ; 40^{\circ} 19^{\prime} \mathrm{W}$ & 21 March 1996 \\
\hline Sotalia fluviatilis (SF009) & 1 & - & 188 & $\begin{array}{l}\text { Praia Grande - } \\
\text { Fundão }\end{array}$ & $20^{\circ} 02^{\prime} \mathrm{S} ; 40^{\circ} 11^{\prime} \mathrm{W}$ & 30 January 1997 \\
\hline Sotalia fluviatilis (SF011) & 1 & $\sigma^{\prime}$ & 175 & $\begin{array}{l}\text { Praia de Itapuã - Vila } \\
\text { Velha }\end{array}$ & $20^{\circ} 22^{\prime} \mathrm{S} ; 40^{\circ} 17^{\prime} \mathrm{W}$ & 6 August 1997 \\
\hline Sotalia fluviatilis (SF012) & 1 & $\sigma$ & 173 & $\begin{array}{l}\text { Praia da Costa - Vila } \\
\text { Velha }\end{array}$ & $20^{\circ} 20^{\prime} \mathrm{S} ; 40^{\circ} 16^{\prime} \mathrm{W}$ & $\begin{array}{l}24 \text { November } \\
1997\end{array}$ \\
\hline Sotalia fluviatilis (SF013) & 1 & $\sigma^{\prime}$ & 170 & $\begin{array}{l}\text { Ponta da Fruta - Vila } \\
\text { Velha }\end{array}$ & $20^{\circ} 31^{\prime} \mathrm{S} ; 40^{\circ} 22^{\prime} \mathrm{W}$ & 25 July 2000 \\
\hline Sotalia fluviatilis (SF014) & 1 & - & - & $\begin{array}{l}\text { Praia de Itapuã - Vila } \\
\text { Velha }\end{array}$ & $20^{\circ} 22^{\prime} \mathrm{S} ; 40^{\circ} 17^{\prime} \mathrm{W}$ & 28 February 2001 \\
\hline Tursiops truncatus 2 & 1 & - & - & Guarapari & $20^{\circ} 39^{\prime} \mathrm{S} ; 40^{\circ} 27^{\prime} \mathrm{W}$ & 23 April 1984 \\
\hline
\end{tabular}

(1) Siciliano and Lodi, 1989, (2) Barros, 1991, (3) Geise and Borobia, 1987, (4) Siciliano, 1994, (5) Ramos et al., 1994. 
region and likely reflects the low monitoring effort on the fishing ports and beaches. Systematic beach surveys and monitoring of fishing fleet are clearly needed to properly evaluate the effects of fisheries on the coastal cetacean species inhabiting this area.

\section{Acknowledgements}

We kindly thank the "Environmental Police" from Espírito Santo State for informing us about the strandings. We also thank César Meyer Musso, Margareth Peixoto Maia, Márcio Amorim Efe, Francisco Pedro da Fonseca Neto and Benedito Valadares, all scientists at AVIDEPA, for helping in strandings. We warmly acknowledge Dra Ana Paula Madeira Di Beneditto for the guidance during this work and Dr Nélio B. Barros for his technical support. Two anonymous referees provided valuable comments on the early drafts of this manuscript.

\section{References}

Barbosa, L.A., Netto, R.F., Moraes, C.S. and Barros, N.B. (2000) Registros de cetáceos para o litoral do Espírito Santo, Brasil. Page 29 in Abstracts, V Simpósio de Ecossistemas Brasileiros: Conservação, 10-15 Outubro, Vitória, ES.

Barros, N.B. (1991) Recent cetacean records for Southeastern Brazil. Marine Mammal Science 7(3): 296-306.

Barros, N.B., Barbosa, L.A. and Gasparini, J.L. (1997a) Distribuição e Biologia de Mamíferos Marinhos no Litoral do Espírito Santo, Brasil. Page 72 in Abstracts, II Encontro de Preservação e Pesquisa de Mamíferos Aquáticos do Norte e Nordeste do Brasil - XI Encontro de Zoologia do Nordeste, Fortaleza, Ceará.

Barros, N.B., Barbosa, L.A., Gasparini, J.L., Netto, R.F. and Moraes, C.S. (1997b) Ingestão de plástico como provável causa mortis de uma baleia-piloto-de-peitorais-curtas, Globicephala macrorhynchus Gray, 1846, no litoral do Espírito Santo. Page 336 in Abstracts, VII Congresso Nordestino de Ecologia, 27 Julho - 2 Agosto, Ilhéus, Bahia.

Borobia, M., Siciliano, S., Lodi, L. and Hoek, W. (1991) Distribution of the South American dolphin Sotalia fluviatilis. Canadian Journal of Zoology 69: 1025-1039.

Danilewicz, D., Tavares, M., Bornholdt, R, Trigo, C.C. and Moreno, I.B. (2002). Unusual Record of a humpback whale (Megaptera novaeangliae) incidentally caught with evidence of recent feeding in the waters off Rio Grande do Sul, Southern Brazil. Pages 72-73 in Abstracts, X Reunión de Trabajo de Especialistas en Mamíferos Acuáticos de América del Sur and IV Congreso de la Sociedad Latinoamericana de Especialistas en Mamíferos Acuáticos de América del Sur, 14-19 October 2002. Valdivia, Chile.

Di Beneditto, A.P., Capistrano, L. and Ramos, R. (1990) Captura acidental de pequenos cetáceos na costa dos estados do Rio de Janeiro, Espírito Santo e Bahia, Brasil. Page 42 in Abstracts, IV Reunion de Trabajo de Especialistas en Mamíferos Acuáticos de América del Sur, Valdivia, Chile.

Di Beneditto, A.P., Ramos, R. and Lima, N.R. (1998) Fishing activity in Northern Rio de Janeiro State (Brazil) and its relation with small cetaceans. Brazilian Archives of Biology and Technology 41(3): 296-302.
Di Beneditto, A.P.M., Ramos, R.M.A. and Lima, N.R.W. (2001) Os golfinhos: origem, classificação, captura acidental, hábito alimentar. Editora Cinco Continentes, Porto Alegre, Brazil.

Gasparini, J.L. and Sazima, I. (1996) A stranded melonheaded whale, Peponocephala electra, in southeastern Brazil, with comments on wounds from the cookiecutter shark, Isistius brasiliensis. Marine Mammal Science 12(2): 308-312.

Geise, L. and Borobia, M. (1987) New Brazilian records for Kogia, Pontoporia, Grampus and Sotalia (Cetacea, Physeteridae, Platanistidae, and Delphinidae). Journal of Mammalogy 68(4): 873-5.

Instituto Brasileiro do Meio Ambiente e dos Recursos Naturais Renováveis - IBAMA (2001) Mamíferos Aquáticos do Brasil. Plano de ação. Ed. MMA/IBAMA, Brasília, Brasil.

Lodi, L., Siciliano, S. and Bellini, C. (1996). Ocorrências e conservação de baleias-francas-do-sul, Eubalaena australis, no litoral do Brasil. Papéis Avulsos de Zoologia (São Paulo), 39(17): 307-328.

Lodi, L. and Hetzel, B. (1998) O golfinho-de-dentes-rugosos (Steno bredanensis) no Brasil. Bioikos 12(1): 29-45.

Ott, P.H., Secchi, E.R., Moreno, I.B., Danilewicz, D., Crespo, E.A., Bordino, P., Ramos, R. Di Beneditto, A.P., Bertozzi, C., Bastida, R., Zanelatto, R., Perez, J.E. and Kinas, P.G. (2002) Report of the Working Group on Fishery Interactions. The Latin American Journal of Aquatic Mammals 1 (special issue 1): 55-64.

Perrin, W.F., Donovan, G.P. and Barlow, J. (1994) Gillnets and Cetaceans. Report of the International Whaling Commission (special issue 15).

Pizzorno, J.L.A., Laílson-Brito, Jr., Dorneles, P.R., Azevedo, A.F., Gurgel, I.M.G.N. (1998) Review of strandings and additional information on humpback whales, Megaptera novaeangliae, in Rio de Janeiro, southern Brazilian coast. Report of the International Whaling Commission 47: 653-658.

Ramos, R.M.A., Di Beneditto, A.P.M. and Fernandes, L.C.C. (1994) Relatório, Plano de Conservação e Manejo de Pequenos Cetáceos - Projeto Cetáceos. Fundação Brasileira para a Conservação da Natureza (unpublished).

Ramos, R.M.A, Di Beneditto, A.P., Siciliano, S., Santos, M.C., Zerbini, A.N., Bertozzi, C., Vicente, A., Zampirolli, E., Alvarenga, F.S. and Lima, N.R.W. (2002) Morphology of the franciscana (Pontoporia blainvillei) off Southeastern Brazil: sexual dimorphism, growth and geographic variation. The Latin American Journal of Aquatic Mammals 1 (special issue 1): 129-144.

Secchi, E.R., Zerbini, A.N., Bassoi, M., Dalla Rosa, L., Möller, L.M. and Rocha-Campos, C. (1997) Mortality of franciscanas, Pontoporia blainvillei, in coastal gillnets in Southeastern Brazil: 1994-1995. Report of the International Whaling Commission 47: 635-58.

Secchi, E.R., Ott, P.H. and Danilewicz, D. (2002) Report of the fourth workshop for the coordinated research and conservation of the franciscana dolphin (Pontoporia blainvillei) in the western South Atlantic. The Latin American Journal of Aquatic Mammals 1 (special issue 1): 11-20.

Siciliano, S. (1987) Nota sobre a captura acidental de Megaptera novaeangliae na costa sudeste do Brasil. Page 115 in Anais, II Reunião de Trabalho de Especialistas em Mamíferos Aquáticos da América do Sul, 4-8 August 1986. Rio de Janeiro, Brazil. 
Siciliano, S. (1994) Review of small cetaceans and fishery interactions in coastal waters of Brazil. Reports of the International Whaling Commission (special issue 15): 241-250.

Siciliano, S. and Lodi, L. (1989) Observations of humpback whale, Megaptera novaeangliae, in the Abrolhos Bank, northeastern Brazil, and a summary of records for the Brazilian coast. Parque Nacional Marinho dos
Abrolhos Technical Report. 38pp (unpublished). Siciliano, S., Di Beneditto, A.P., and Ramos, R.M.A. (2002) A toninha, Pontoporia blainvillei (Gervais \& d'Orbigny, 1844) (Mammalia, Cetacea, Pontoporiidae), nos estados do Rio de Janeiro e Espírito Santo, costa sudeste do Brasil: caracterizações dos habitats e fatores de isolamento das populações. Boletim do Museu Nacional, Nova Série, Zoologia, 476: 1-15. 